\title{
A new histological therapeutic classification system to predict eradicated and residual lymph nodes in breast cancer after neoadjuvant chemotherapy
}

\author{
SATOKO MOROHASHI ${ }^{1}$, TADASHI YOSHIZAWA ${ }^{1}$, HIROKO SEINO ${ }^{2}$, HIDEAKI HIRAI $^{1}$, TOSHIHIRO HAGA ${ }^{1}$, \\ RIE OTA $^{1}$, YUNYAN WU ${ }^{1}$, ERI YOSHIDA ${ }^{3}$, KENICHI HAKAMADA ${ }^{3}$ and HIROSHI KIJIMA ${ }^{1}$ \\ Departments of ${ }^{1}$ Pathology and Bioscience, ${ }^{2}$ Radiology and Radiation Oncology and ${ }^{3}$ Gastroenterological Surgery, \\ Hirosaki University Graduate School of Medicine, Hirosaki, Aomori 036-8562, Japan
}

Received November 13, 2015; Accepted December 23, 2015

DOI: $10.3892 /$ or.2016.4635

\begin{abstract}
The indication for neoadjuvant chemotherapy (NAC) has recently broadened to include its use in the treatment of initial stage breast cancer. Axillary lymph node metastasis after NAC in breast cancer is a poor prognostic factor. Thus, the prediction of lymph node metastasis is important to estimate the prognosis of breast cancer patients after NAC. Therefore, we focused on residual carcinoma patterns of primary breast tumors after NAC and examined the correlation between the patterns and lymph node metastasis. In this study, we examined 50 breast cancer specimens and associated dissected lymph nodes after NAC. We divided 40 cases into an eradicated lymph node group and a residual lymph node group to analyze residual carcinoma patterns of primary breast tumors. Residual carcinoma patterns were classified according to the cell density of carcinoma cells: dense, focal/nested and sporadic/in-situ. There were significant differences in residual carcinoma patterns $(\mathrm{P}<0.01)$ among the three pattern groups. There was a high incidence of dense patterns in the residual lymph node group and a high incidence of sporadic/in-situ patterns in the eradicated lymph node group. Analysis of residual carcinoma patterns of primary breast tumors and clinicopathological factors demonstrated that there were significant differences in tumor reduced ratio on $\mathrm{CT}(\mathrm{P}<0.001)$, primary tumor area before NAC $(\mathrm{P}<0.01)$, primary tumor area after NAC $(\mathrm{P}<0.00001)$, intrinsic subtype $(\mathrm{P}<0.01), \mathrm{Ki}-67$ labeling index $(\mathrm{P}<0.01)$, histological grade $(\mathrm{P}<0.05)$ and mitotic count
\end{abstract}

Correspondence to: Dr Satoko Morohashi, Department of Pathology and Bioscience, Hirosaki University Graduate School of Medicine, 5 Zaifu-cho, Hirosaki, Aomori 036-8562, Japan

E-mail: msatoko@hirosaki-u.ac.jp

Abbreviations: NAC, neoadjuvant chemotherapy

Key words: breast cancer, neoadjuvant chemotherapy, therapeutic effect, lymph node metastasis
$(\mathrm{P}<0.01)$ between the dense and non-dense groups. Therefore, our results suggest that the residual carcinoma pattern is useful for predicting eradicated or residual lymph nodes and the malignant potential in breast cancer after NAC.

\section{Introduction}

A complete pathologic response of breast cancer after neoadjuvant chemotherapy (NAC) appears to be particularly favorable at 5 years in patients with the least evidence of a tumor in the breast or lymph nodes after therapy $(1,2)$. The National Surgical Adjuvant Breast and Bowel Project trials distinguished between the absence and presence of residual invasive carcinoma in the breast and noted that long-term outcome was also dependent on the extent of lymph node involvement (1). Axillary lymph node metastasis after NAC in breast cancer is a poor prognostic factor (2). Residual micro-metastatic disease in the axillary lymph nodes after NAC is a worse prognostic factor than negative nodes in breast cancer (3). Therefore, prediction of lymph node metastasis is important for prognosis and choosing an optimal therapeutic strategy for the treatment of breast cancer after NAC.

Previous reports have described predictive factors of complete pathological response in primary breast tumors after NAC (4). Judgement of pathological therapeutic effects of breast cancer after NAC vary among studies (5-12). However, no article has described residual carcinoma patterns of the primary breast tumor to predict the presence or absence of lymph node metastasis. Therefore, we devised a classification system of three residual carcinoma patterns using a simple method based on cell density: dense, focal/nested, and sporadic/in-situ. In this study, we examined 50 cases of breast cancer and associated dissected lymph nodes after NAC and divided the cases into an eradicated lymph node group (14 cases), a residual lymph node group (26 cases), and a no change lymph node group (10 cases) to compare differences in the three residual carcinoma patterns in primary breast tumor between the eradicated lymph node and residual lymph node groups. Furthermore, we analyzed differences in clinicopathological factors in residual carcinoma patterns of the primary breast tumors. 


\section{Materials and methods}

Patient samples. We retrospectively evaluated computed tomography (CT) or positron emission tomography-computed tomography (PET-CT) scans of 50 surgically resected breast cancer lesions taken before and after NAC between 2006 and 2015 at Hirosaki University Hospital (Hirosaki, Aomori, Japan). Informed consent was obtained from each patient regarding the use of clinical records and pathological specimens. Twenty-nine patients underwent total mastectomy, and 21 underwent partial mastectomy. Sentinel node biopsy was performed for 2 cases, level I lymph node dissection for 48 cases, level II lymph node dissection for 23 cases, level III lymph node dissection for 3 cases, and Rotter dissection for 2 cases. Chemotherapy regimens are shown in Table I. The chemotherapy regimens were performed as follows: $\mathrm{AC}$ (doxorubicin + cyclophosphamide), 4 cases $(8 \%) ; \mathrm{AC} \rightarrow \mathrm{T}$ (doxorubicin + cyclophosphamide followed by taxane), 24 cases (48\%); AC $\rightarrow \mathrm{T}+$ HER (doxorubicin + cyclophosphamide followed by taxane + trastuzumab), 12 cases $(24 \%)$; EC (epirubicin hydrochloride + cyclophosphamide hydrate), 2 cases (4\%); EC $\rightarrow \mathrm{T}$ (epirubicin hydrochloride + cyclophosphamide hydrate followed by taxane), 7 cases (14\%); and TC (docetaxel + cyclophosphamide hydrate), 1 case $(2 \%)$. The mean number of cycles per regimen was 7.66 (range, 4-8). Union for International Cancer Control (UICC) stages of before and after NAC are shown in Table II. UICC clinical stages (cStage) before NAC were as follows: cStage 0, 0 cases ( $0 \%)$; cStage I, 2 cases (4\%); cStage II, 26 cases (52\%); and cStage III, 22 cases (44\%). UICC cStages after NAC were as follows: cStage 0,0 cases $(0 \%)$; cStage I, 16 cases $(32 \%)$; cStage II, 24 cases (48\%); and cStage III, 9 cases (18\%). UICC pathological stages (pStage) after NAC were as follows: pStage 0,4 cases (8\%); pStage I, 13 cases (26\%); pStage II, 18 (36\%); and pStage III, 15 cases $(30 \%)$.

Pathological examinations of the primary tumors. Partial mastectomy specimens were sliced into $5 \mathrm{~mm}$ sections and total mastectomy specimens, which included the maximal tumor sectioned surface, were sliced as much as possible to distinguish the lesion. For histopathological examination, breast cancer specimens were routinely formalin-fixed, paraffin-embedded, thinly sectioned, and stained with hematoxylin and eosin. Carcinoma lesions were histologically graded according to the Bloom-Richardson system (13). Tumor maximal invasion diameters were measured and the extent of lymphatic invasion, venous invasion and intraductal component was evaluated. The status of the estrogen receptor (ER), progesterone receptor (PgR), and HER2 was immunohistochemically detected. ER/PR expression was defined as positive when $\geq 10 \%$ of nuclei in the total tumor cells were stained. As to HER2: 1, negative, 2, uncertain, and 3, positive. Cancers with an HER2 score of 2+ were additionally evaluated using dual-color in situ hybridization. In this study, breast cancer was classified into four groups as follows: luminal A (ER and/PR-positive/HER2-negative/low Ki-67), luminal B (ER- and/or PR-positive/HER2-negative/high Ki-67), luminal B (ER- and/or PR-positive/HER2 overexpression/any Ki-67), HER2 (ER and PR absent/HER2 overexpression), and triple-negative (ER and PR absent/HER2-negative) (14).
Table I. Chemotherapy regimens and cycles.

\begin{tabular}{lc}
\hline Chemotherapy regimen & $\mathrm{n}(\%)$ \\
\hline $\mathrm{AC}$ & $4(8)$ \\
$\mathrm{AC} \rightarrow \mathrm{T}$ & $24(48)$ \\
$\mathrm{AC} \rightarrow \mathrm{T}+\mathrm{HER}$ & $12(24)$ \\
$\mathrm{EC}$ & $2(4)$ \\
$\mathrm{EC} \rightarrow \mathrm{T}$ & $7(14)$ \\
$\mathrm{TC}$ & $1(2)$ \\
Mean cycles (range) & $7.66(4-8)$ \\
\hline
\end{tabular}

$\mathrm{AC}$, doxorubicin + cyclophosphamide; $\mathrm{AC} \rightarrow \mathrm{T}$, doxorubicin + cyclophosphamide followed by taxane; $\mathrm{AC} \rightarrow \mathrm{T}+\mathrm{HER}$, doxorubicin + cyclophosphamide followed by taxane + trastuzumab; EC, epirubicin hydrochloride + cyclophosphamide hydrate; $\mathrm{EC} \rightarrow \mathrm{T}$, epirubicin hydrochloride + cyclophosphamide hydrate followed by taxane; TC, docetaxel + cyclophosphamide hydrate.

Table II. UICC stage before and after NAC.

\begin{tabular}{lccc}
\hline & $\begin{array}{c}\text { UICC cStage } \\
\text { before NAC } \\
\mathrm{n}(\%)\end{array}$ & $\begin{array}{c}\text { UICC cStage } \\
\text { after NAC } \\
\mathrm{n}(\%)\end{array}$ & $\begin{array}{c}\text { UICC pStage } \\
\text { after NAC } \\
\mathrm{n}(\%)\end{array}$ \\
\hline Stage 0 & $0(0)$ & $0(0)$ & $4(8)$ \\
Stage I & $2(4)$ & $16(32)$ & $13(26)$ \\
Stage II & $26(52)$ & $24(48)$ & $18(36)$ \\
Stage III & $22(44)$ & $9(18)$ & $15(30)$ \\
\hline
\end{tabular}

UICC, Union for International Cancer Control; cStage, clinical stage; pStage, pathological stage; NAC, neoadjuvant chemotherapy.

Residual carcinoma patterns. Whole sections examined in this study were divided into three residual carcinoma patterns: dense (tumor cell density remained in a very high state), focal/ nested (tumor cells disappeared focally with fibrosis or granulation tissue or macrophage infiltration, or elastic fiber within the tumor area), and sporadic/in situ (a few cancer nests remained or in situ lesion only remained). If a biopsy was performed before chemotherapy, we compared the surgically resected specimen with its biopsy results as much as possible (Fig. 1). Forty biopsies of primary tumors before NAC were evaluated in this study. Focal/nested and sporadic/in-situ patterns were included in the non-dense group when we analyzed residual carcinoma patterns and clinicopathological factors.

Pathological examination of lymph nodes. We chose lymph nodes from 14 cases that were examined by fine needle aspiration biopsy cytology or core needle biopsy before NAC. Five cases that were positive by lymph node biopsy before NAC and in which lymph node metastasis disappeared after NAC were histologically examined in detail. Lymph nodes that were more than $5 \%$ fibrotic were defined as eradicated lymph nodes. We did not consider fibrosis of the lymph node capsule. We chose lymph node specimens with irregular fibrosis. The sections were scanned using light microscopy at a low magnification (x12.5 or $x 40)$. The composition of the image was carried out 

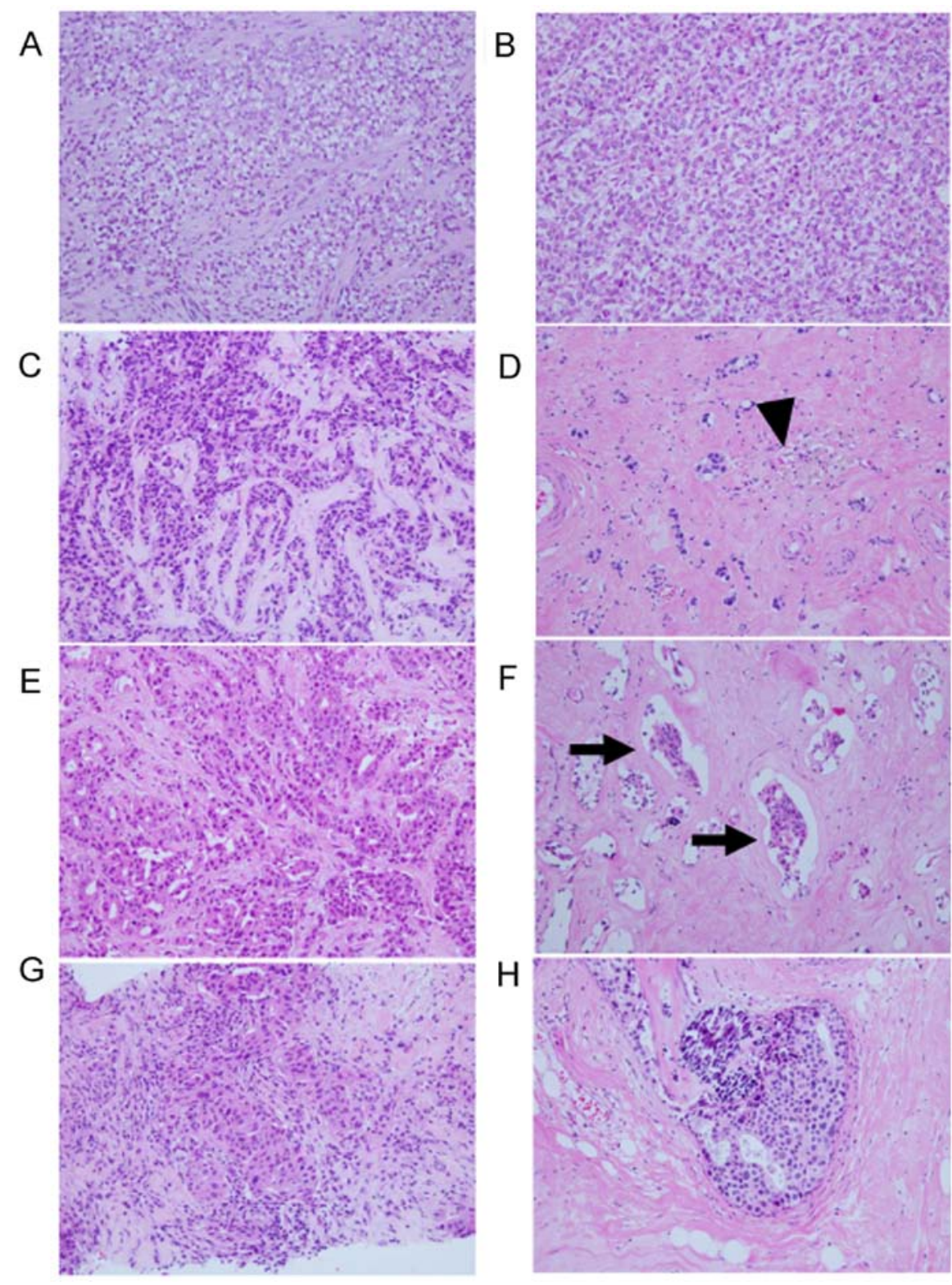

Figure 1. (A, C, E and G) Core needle biopsy before neoadjuvant chemotherapy (NAC). (B, D, F and H) Surgical specimens of the same case as the left photograph. (A and B) This case was classified as dense since cancer cell density was very high in the surgical specimen. Histologically, there was no therapeutic effect observed. (C and D) This case was classified as focal/nested. Hemosiderin laden macrophages were noted in the stroma (arrowhead), but cancer cell density was still high. (E and F) This case was classified as sporadic as a few cancer nests were present (arrows). (G and H) This case was classified as in situ as only a non-invasive ductal component was present.

in Adobe Photoshop (Adobe ${ }^{\circledR}$ Photoshop $^{\circledR}$ CS2 Windows ${ }^{\circledR}$; USA). The extent of fibrosis within the lymph nodes was measured using ImageJ software (15). Three cases that were negative by lymph node biopsy before NAC and negative for lymph node metastasis after NAC were also histologically examined in detail. 'No change' lymph nodes included those with few hemosiderin laden macrophages and no fibrosis.

Division of the eradicated and residual lymph node groups. Fifty breast cancer cases after NAC were divided into an eradiated lymph node group, a residual lymph node group, and a no change lymph node group. The no change lymph node cases were excluded in this study. Differences in the three residual carcinoma patterns (dense, focal/nested, and sporadic/in-situ) between the eradiated lymph node and residual lymph node groups were examined.
Residual carcinoma patterns and clinicopathological factors. Residual carcinoma patterns were divided into two groups, a dense group and a non-dense group. Focal/nested and sporadic/in-situ patterns were defined as the non-dense group. Differences in the following clinicopathological factors between the dense and non-dense groups were examined: trastuzumab administration, reduced ratio on CT, primary tumor area before/after NAC on CT, intrinsic subtype, ER status, PgR status, HER2 status, Ki-67 labeling index, primary tumor pathological diameter, lymphatic invasion, venous invasion, histological grade, nuclear atypia, mitotic count, tubular formation, and extent of intraductal components. Primary breast tumor areas before and after NAC were calculated using DICOM data on CT images. The primary tumor area was calculated three times for each case and the mean value was used for analysis. The reduced ratio was calculated using the 
Table III. Histological findings of the surgical resection specimens after neoadjuvant chemotherapy.

\begin{tabular}{|c|c|}
\hline Features & $\mathrm{n}=50(100 \%)$ \\
\hline \multicolumn{2}{|l|}{ Intrinsic subtype } \\
\hline Luminal A & $13(26)$ \\
\hline Luminal B & $28(56)$ \\
\hline HER2 & $4(8)$ \\
\hline Triple-negative & $5(10)$ \\
\hline \multicolumn{2}{|l|}{ Histology } \\
\hline Ductal carcinoma in situ & $4(4)$ \\
\hline Invasive ductal carcinoma & $38(76)$ \\
\hline Invasive lobular carcinoma & $4(8)$ \\
\hline Invasive micropapillary carcinoma & $1(2)$ \\
\hline Mucinous carcinoma & $2(4)$ \\
\hline Intracystic papillary carcinoma & $1(2)$ \\
\hline \multicolumn{2}{|l|}{ Lymph node status } \\
\hline Negative & $26(52)$ \\
\hline Positive & $24(48)$ \\
\hline \multicolumn{2}{|l|}{ Lymphatic invasion } \\
\hline Negative & $31(62)$ \\
\hline Positive & $19(38)$ \\
\hline \multicolumn{2}{|l|}{ Venous invasion } \\
\hline Negative & $46(92)$ \\
\hline Positive & $4(8)$ \\
\hline \multicolumn{2}{|l|}{ Histological grade } \\
\hline I & $15(30)$ \\
\hline II & $27(54)$ \\
\hline III & $8(16)$ \\
\hline \multicolumn{2}{|l|}{ Nuclear atypia } \\
\hline 1 & $3(6)$ \\
\hline 2 & $27(54)$ \\
\hline 3 & $20(40)$ \\
\hline \multicolumn{2}{|l|}{ Mitotic count } \\
\hline 1 & $32(64)$ \\
\hline 2 & $14(28)$ \\
\hline 3 & $4(8)$ \\
\hline \multicolumn{2}{|l|}{ Tubular formation } \\
\hline 1 & $1(2)$ \\
\hline 2 & $23(46)$ \\
\hline 3 & $26(52)$ \\
\hline \multicolumn{2}{|l|}{ Extensive intraductal component } \\
\hline Negative & $38(76)$ \\
\hline Positive & $12(24)$ \\
\hline
\end{tabular}

mean area of the primary breast tumor before and after NAC as follows: Reduced ratio $=(1-$ mean area after NAC/mean area before NAC) x 100 .

Statistical analyses. Statistical comparisons between two groups were analyzed using the Pearson's Chi-square test for categorical data and the Wilcoxon rank sum test for continuous data. Differences were considered to be statistically significant at a $p$-value of $<0.05$. An adjusted residue of \pm 2 or more was
Table IV. Residual carcinoma patterns in primary breast tumors and lymph node metastasis.

\begin{tabular}{|c|c|c|c|}
\hline $\begin{array}{l}\text { Residual } \\
\text { carcinoma } \\
\text { pattern }\end{array}$ & $\begin{array}{c}\text { Residual } \\
\text { lymph node } \\
\text { group } \\
(\mathrm{n}=26)(100 \%) \\
\mathrm{n}(\%)\end{array}$ & $\begin{array}{c}\text { Eradicated } \\
\text { lymph node } \\
\text { group } \\
(\mathrm{n}=14)(100 \%) \\
\mathrm{n}(\%)\end{array}$ & $\mathrm{P}$-value \\
\hline Dense & $11(42.3)^{\mathrm{a}}$ & $1(7.1)^{b}$ & 0.003 \\
\hline Focal/nested & $15(57.7)$ & $9(64.3)$ & \\
\hline Sporadic/in-situ & $0(0)^{\mathrm{b}}$ & $4(28.6)^{\mathrm{a}}$ & \\
\hline
\end{tabular}

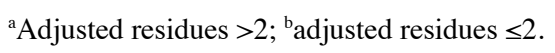

considered to be significant. All statistical evaluations were performed using R (http://www.r-project.org) and IBM $^{\circledR}$ SPSS ${ }^{\circledR}$ Statistics version 22 (IBM Corporation, Armonk, NY, USA) software.

\section{Results}

Tumor characteristics. Tumor characteristics are shown in Table III. Intrinsic subtype included: luminal A, 13 cases (26\%); luminal B, 28 cases $(56 \%)$; HER2 type, 4 cases $(8 \%)$ and triple-negative, 5 cases $(10 \%)$. Histology included: ductal carcinoma in situ, 4 cases (4\%); invasive ductal carcinoma, 38 cases $(76 \%)$; invasive lobular carcinoma, 4 cases $(8 \%)$; invasive micropapillary carcinoma, 1 case $(2 \%)$; mucinous carcinoma, 2 cases (4\%) and intracystic papillary carcinoma, 1 case $(2 \%)$. Lymph node status included: negative, 26 cases (52\%) and positive, 24 cases (48\%). Lymphatic invasion included: negative, 31 cases $(62 \%)$ and positive, 19 cases (38\%). Venous invasion included: negative, 46 cases $(92 \%)$ and positive, 4 cases $(8 \%)$. Histological grade included: I, 15 cases (30\%); II, 27 cases (54\%) and III, 8 cases (16\%). Nuclear atypia included: 1,3 cases (6\%); 2, 27 cases (54\%) and 3, 20 cases (40\%). Mitotic count included: 1, 32 cases (64\%); 2, 14 cases (28\%) and 3, 4 cases (8\%). Tubular formation included: 1, 1 case (2\%); 2, 23 cases (46\%) and 3, 26 cases (52\%). Extensive intraductal components included: negative, 38 cases $(76 \%)$ and positive, 12 cases $(24 \%)$.

Eradicated lymph node. Fine needle aspiration biopsy cytology or core needle biopsy of five eradicated lymph node cases performed before NAC showed fibrosis within the lymph nodes. The percentage of fibrosis of the five lymph nodes was $34.6,19.8,14.0,9.6$, and $5.3 \%$, respectively. A representative specimen of an eradicated lymph node with $19.8 \%$ fibrosis within the lymph node is shown in Fig. 2. Three cases with lymph nodes negative before NAC and no lymph node metastasis after NAC had only a few hemosiderin laden macrophages and no fibrosis (Fig. 3). These lymph nodes were classified as no change lymph nodes.

Residual carcinoma patterns and lymph node metastasis of the eradicated and residual lymph node groups. There were significant differences in the residual patterns $(\mathrm{P}=0.0034)$ 
A

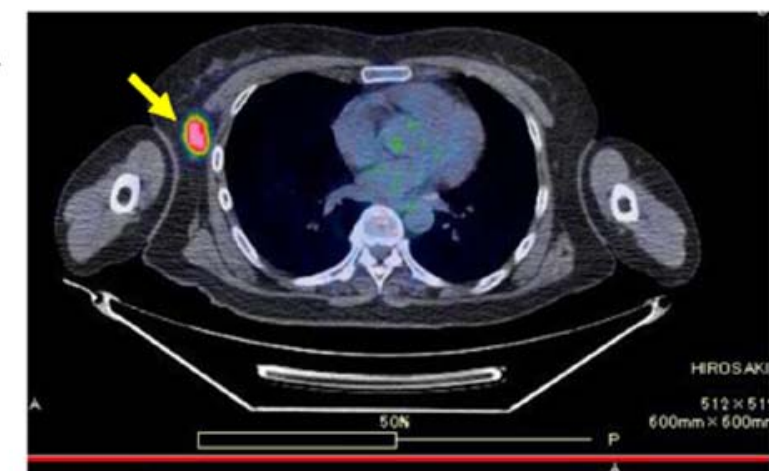

B

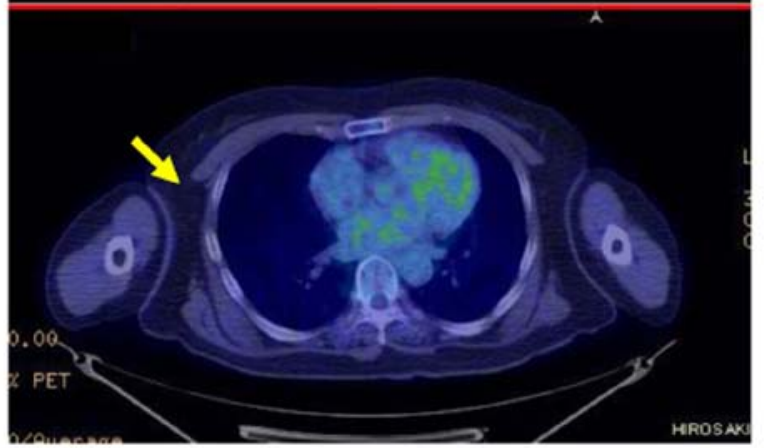

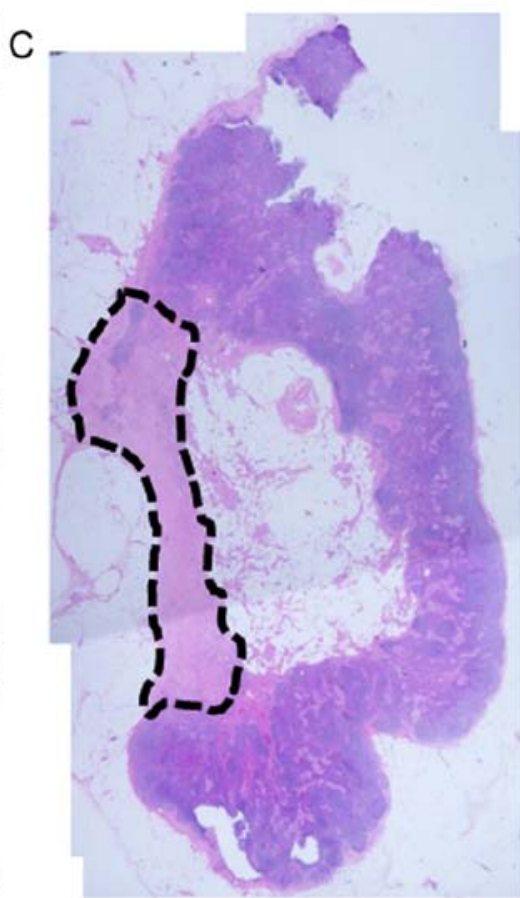

Figure 2. (A) ${ }^{18} \mathrm{~F}$-fluorodeoxyglucose positron emission tomography/computed tomography (PET-CT) before neoadjuvant chemotherapy (NAC). Lymph node metastasis was observed using PET-CT. Lymph node metastasis was confirmed using core needle biopsy before NAC in this case. (B) PET-CT after NAC, no lymph node metastasis was observed on PET-CT. (C) Irregular fibrosis of $19.8 \%$ is noted within the lymph node, as measured using ImageJ software.

A

B
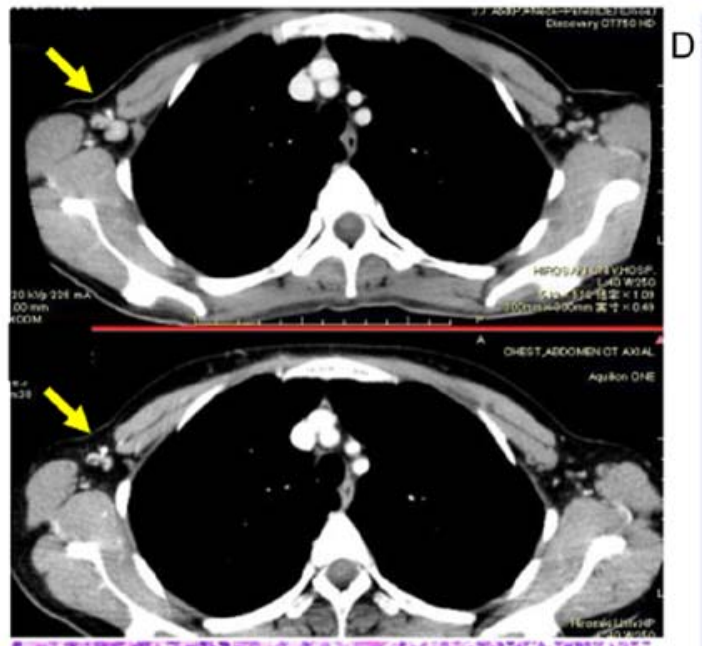

C
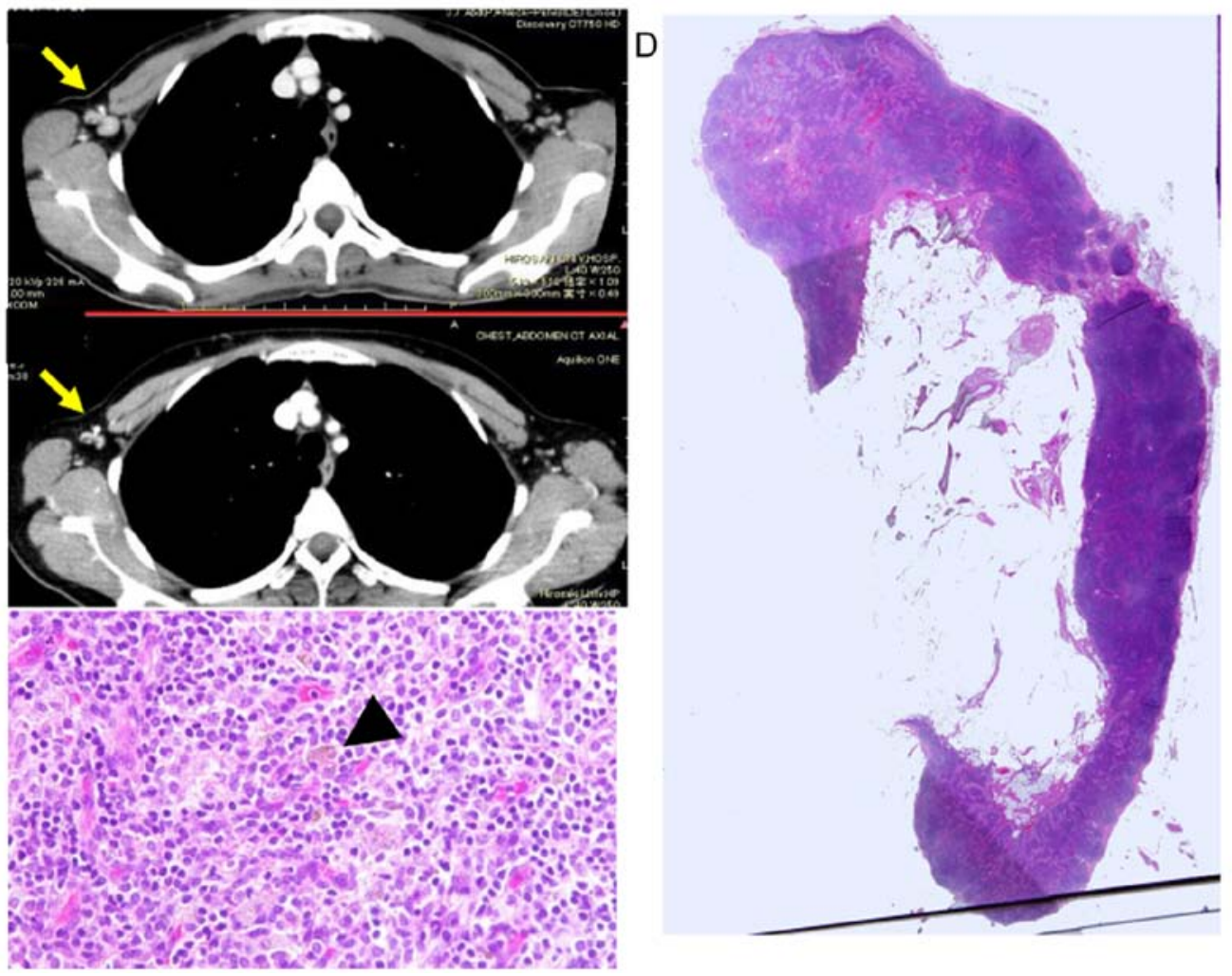

Figure 3. (A) Enhanced computed tomography (CT) before neoadjuvant chemotherapy (NAC). This case was suspected as lymph node metastasis using imaging. There was no carcinoma metastasis by fine needle aspiration biopsy cytology before NAC. (B) Enhanced CT after NAC. The lymph node was smaller than before NAC. (C) A few hemosiderin laden macrophages are noted in the negative lymph node (arrowhead). (D) There is no apparent fibrosis in the lymph node. This lymph node was classified as a 'no change' lymph node.

between the eradicated and residual lymph node groups as many of the residual lymph nodes had dense patterns (adjusted residue +2.3 ) and many of the eradicated lymph nodes had sporadic/in-situ patterns (adjusted residue +2.9) (Table IV). 
Table V. Clinicopathological factors of the non-dense and dense group.

\begin{tabular}{|c|c|c|c|}
\hline & $\begin{array}{c}\text { Non-dense } \\
(\mathrm{n}=35)(100 \%) \\
\mathrm{n}(\%)\end{array}$ & $\begin{array}{c}\text { Dense } \\
(\mathrm{n}=15)(100 \% \\
\mathrm{n}(\%)\end{array}$ & P-va \\
\hline Age (mean, years) & 50.6 & 49.0 & 0.567 \\
\hline \multicolumn{4}{|l|}{ Trastuzumab } \\
\hline On & $10(28.6)$ & $2(13.3)$ & 0.304 \\
\hline Off & $25(71.4)$ & $13(86.7)$ & \\
\hline Reduced ratio & 0.089 & 0.496 & $<0.001^{\mathrm{a}}$ \\
\hline Area before NAC & $336.5 \mathrm{~mm}^{2}$ & $1014.0 \mathrm{~mm}^{2}$ & $0.003^{\mathrm{a}}$ \\
\hline Area after & $33.6 \mathrm{~mm}^{2}$ & $213.7 \mathrm{~mm}^{2}$ & $<0.00001$ \\
\hline \multicolumn{4}{|l|}{ Intrinsic subtype } \\
\hline Luminal A & $10(28.6)$ & $3(20.0)$ & $0.004^{\mathrm{a}}$ \\
\hline Luminal B & $21(60.0)$ & $7(46.7)$ & \\
\hline HER2 & $4(11.4)$ & $0(0)$ & \\
\hline Triple-negative & $0(0)^{\mathrm{c}}$ & $5(33.3)^{b}$ & \\
\hline \multicolumn{4}{|l|}{ ER status } \\
\hline Positive & $31(88.6)$ & $10(66.7)$ & 0.105 \\
\hline Negative & $4(11.4)$ & $5(33.3)$ & \\
\hline \multicolumn{4}{|l|}{ PgR status } \\
\hline Positive & $20(57.1)$ & $6(40.0)$ & 0.266 \\
\hline Negative & $15(42.9)$ & $9(60.0)$ & \\
\hline \multicolumn{4}{|l|}{ HER2 status } \\
\hline Positive & $14(40.0)$ & $2(13.3)$ & 0.098 \\
\hline Negative & $21(60.0)$ & $13(86.7)$ & \\
\hline Ki-67 labeling index & 5 & 50 & $0.006^{\mathrm{a}}$ \\
\hline Tumor diameter $(\mathrm{mm})$ & 11 & 30 & $0.010^{\mathrm{a}}$ \\
\hline \multicolumn{4}{|l|}{ Lymphatic invasion } \\
\hline Positive & $11(31.4)$ & $8(53.3)$ & 0.143 \\
\hline Negative & $24(68.6)$ & $7(46.7)$ & \\
\hline \multicolumn{4}{|l|}{ Venous invasion } \\
\hline Positive & $1(2.9)$ & $3(20.0)$ & 0.075 \\
\hline Negative & $34(97.1)$ & $12(80.0)$ & \\
\hline \multicolumn{4}{|l|}{ Histological grade } \\
\hline I & $14(40.0)^{\mathrm{b}}$ & $1(6.7)^{\mathrm{c}}$ & $0.016^{\mathrm{a}}$ \\
\hline II & $18(51.4)$ & $9(60.0)$ & \\
\hline III & $3(8.6)^{\mathrm{c}}$ & $5(33.3)^{b}$ & \\
\hline \multicolumn{4}{|l|}{ Nuclear atypia } \\
\hline 1 & $3(8.6)$ & $0(0)$ & 0.616 \\
\hline 2 & $19(54.3)$ & $8(53.3)$ & \\
\hline 3 & $13(37.1)$ & $7(46.7)$ & \\
\hline \multicolumn{4}{|l|}{ Mitotic count } \\
\hline 1 & $26(74.3)^{\mathrm{b}}$ & $6(40.0)^{\mathrm{c}}$ & $0.004^{\mathrm{a}}$ \\
\hline 2 & $9(25.7)$ & $5(33.3)$ & \\
\hline 3 & $0(0)^{\mathrm{c}}$ & $4(26.7)^{b}$ & \\
\hline \multicolumn{4}{|l|}{ Tubular formation } \\
\hline 1 & $1(2.9)$ & $0(0)$ & $0.027^{\mathrm{a}}$ \\
\hline 2 & $20(57.1)^{\mathrm{b}}$ & $3(20.0)^{\mathrm{c}}$ & \\
\hline 3 & $14(40.0)^{\mathrm{c}}$ & $12(80.0)^{\mathrm{b}}$ & \\
\hline
\end{tabular}

Table V. Continued.

\begin{tabular}{lccc}
\hline & $\begin{array}{c}\text { Non-dense } \\
(\mathrm{n}=35)(100 \%) \\
\mathrm{n}(\%)\end{array}$ & $\begin{array}{c}\text { Dense } \\
(\mathrm{n}=15)(100 \%) \\
\mathrm{n}(\%)\end{array}$ & P-value \\
& & & \\
EIC & & & \\
Positive & $9(25.7 \%)$ & $3(20.0 \%)$ & 1.00 \\
Negative & $26(74.3 \%)$ & $12(80.0 \%)$ &
\end{tabular}

${ }^{\mathrm{a}} \mathrm{P}<0.05$. ${ }^{\mathrm{b}}$ adjusted residue $>2$, ${ }^{\mathrm{c}}$ adjusted residue $<-2$. NAC, neoadjuvant chemotherapy; ER, estrogen receptor; PgR, progesterone receptor; EIC, extensive intraductal component. All data values are expressed in $\mathrm{n}(\%)$ except for age, reduced ratio, area before/after NAC, Ki-67 labeling index and tumor diameter.

Residual carcinoma patterns and clinicopathological factors. Clinicopathological factors of the non-dense and dense group are shown in Table V. The dense group had a higher reduced ratio $(\mathrm{P}<0.001)$, larger area before NAC $(\mathrm{P}=0.003)$, and larger area after NAC $(\mathrm{P}<0.00001)$, as compared with the non-dense group. There were significant differences in the intrinsic subtype $(\mathrm{P}=0.004)$ between groups. Particularly, there were a larger number of triple-negative cases in the dense group (adjusted residue +3.6). The Ki-67 labeling index was higher in the dense group $(\mathrm{P}=0.006)$ than that in the non-dense group. Pathological tumor diameter was larger in the dense group $(\mathrm{P}=0.010)$ than that in the non-dense group. The histological grade $(\mathrm{P}=0.016)$ and mitotic count $(\mathrm{P}=0.004)$ were higher, and tubular formation $(\mathrm{P}=0.027)$ was less frequent in the dense group than that in the non-dense group. There were no significant differences in age $(\mathrm{P}=0.567)$, trastuzumab administration $(\mathrm{P}=0.304), \mathrm{ER}(\mathrm{P}=0.105), \mathrm{PgR}(\mathrm{P}=0.266)$, HER2 status $(\mathrm{P}=0.098)$, lymphatic invasion $(\mathrm{P}=0.143)$, venous invasion $(\mathrm{P}=0.075)$, nuclear atypia $(\mathrm{P}=0.616)$, or extensive intraductal component $(\mathrm{P}=1.00)$ between the dense and non-dense groups.

\section{Discussion}

The results of this study suggest that residual carcinoma patterns (dense, focal/nested, and sporadic/in-situ) in primary breast cancer after NAC are correlated with lymph node metastasis status. There was a high incidence of dense patterns in the residual lymph node group and a high incidence of sporadic/ in-situ patterns in the eradicated lymph node group. The dense group had malignant potential of breast cancer after NAC when compared with the non-dense group. The tumor areas before and after NAC were larger in the dense group than that in the non-dense group. The Ki-67 labeling index, histological grade, mitotic count, and extent of tubular formation were higher in the dense group than these parameters in the non-dense group. Interestingly, the tumor reduced ratio after NAC was larger in the dense group than that in the non-dense group.

The 'dense' residual carcinoma pattern was associated with the potential for residual carcinoma in the lymph nodes. No previous report has predicted lymph node metastasis in breast cancer after NAC using residual carcinoma patterns. The Miller and Payne system is used to assess the response to chemotherapy based on a 5-point histological grading system 
of fundamental features that include reduction in tumor cellularity and comparisons with pre-treatment core biopsies. This grading system is correlated with overall survival and disease-free survival (16). The Millar and Payne system and our proposed histological classification system suggest that tumor cellularity is the most important characteristic to assess the response to NAC in breast cancer. Rajan et al also reported that cellularity was useful to assess the pathologic responses of breast cancer to chemotherapy (17). A 'dense' residual carcinoma pattern has malignant potential after NAC in breast cancer. Resistance of tumor cells to chemotherapy is suggested by a high Ki-67 labeling index, high mitotic count, and large tumor area. A dense pattern is associated with a high histological grade due to a high mitotic count and less tubular formation.

A residual carcinoma pattern is based on a very simple criteria of tumor cell density and change in histological degeneration but is not dependent on tumor area before NAC. Therefore, it is easy to predict the therapeutic effect using histological diagnosis without image analysis. It is possible to recognize the presence of a dense pattern using core needle biopsy after NAC; therefore, it may be possible to predict lymph node metastasis before surgery by core needle biopsy using our proposed residual carcinoma patterns.

This new classification system considers only three patterns. Although a relatively small number of cases were analyzed, the focal/nested pattern demonstrated wide range chemotherapy responses. By analyzing a larger number of cases in the future, focal/nested cases can be divided into two groups, i.e., focal/nested low and high. This study was a small-scale analysis carried out at a single institution, and there was difficulty with the unified chemotherapy regimen. Before NAC, the prediction of the presence or absence of lymph node metastasis was dependent only on imaging and sometimes after starting treatment. The findings of this study were derived from detailed histopathological analyses of primary breast tumors and associated dissected lymph nodes after NAC. Therefore, these results were realistic and may be useful to predict lymph node metastasis after NAC. Further identification of lymph node metastasis predictors is expected by the additional accumulation of cases in the future.

The results of this study suggest that a residual carcinoma pattern may be predictive of lymph node metastasis after NAC in breast cancer and a dense residual carcinoma pattern of a primary breast tumor after NAC may be an indicator of therapeutic resistance to NAC.

\section{Acknowledgements}

This study was supported by Grants-in-Aid for Science from the Ministry of Education, Culture, Sports, Science, and Technology of Japan; a Grant for Hirosaki University Institutional Research; and the Fund for the Promotion of International Scientific Research.

\section{References}

1. Fisher ER, Wang J, Bryant J, Fisher B, Mamounas E and Wolmark N: Pathobiology of preoperative chemotherapy: Findings from the National Surgical Adjuvant Breast and Bowel (NSABP) protocol B-18. Cancer 95: 681-695, 2002.
2. Kuerer HM, Sahin AA, Hunt KK, Newman LA, Breslin TM, Ames FC, Ross MI, Buzdar AU, Hortobagyi GN and Singletary SE: Incidence and impact of documented eradication of breast cancer axillary lymph node metastases before surgery in patients treated with neoadjuvant chemotherapy. Ann Surg 230: 72-78, 1999.

3. Klauber-DeMore N, Ollila DW, Moore DT, Livasy C, Calvo BF, Kim HJ, Dees EC, Sartor CI, Sawyer LR, Graham M II, et al: Size of residual lymph node metastasis after neoadjuvant chemotherapy in locally advanced breast cancer patients is prognostic. Ann Surg Oncol 13: 685-691, 2006.

4. Kuerer HM, Newman LA, Smith TL, Ames FC, Hunt KK, Dhingra K, Theriault RL, Singh G, Binkley SM, Sneige N, et al: Clinical course of breast cancer patients with complete pathologic primary tumor and axillary lymph node response to doxorubicin-based neoadjuvant chemotherapy. J Clin Oncol 17: 460-469, 1999

5. Mamounas EP, Anderson SJ, Dignam JJ, Bear HD, Julian TB Geyer CE Jr, Taghian A, Wickerham DL and Wolmark N: Predictors of locoregional recurrence after neoadjuvant chemotherapy: Results from combined analysis of National Surgical Adjuvant Breast and Bowel Project B-18 and B-27. J Clin Oncol 30: 3960-3966, 2012.

6. Huang EH, Strom EA, Perkins GH, Oh JL, Chen AM, Meric-Bernstam F, Hunt KK, Sahin AA, Hortobagyi GN and Buchholz TA: Comparison of risk of local-regional recurrence after mastectomy or breast conservation therapy for patients treated with neoadjuvant chemotherapy and radiation stratified according to a prognostic index score. Int J Radiat Oncol Biol Phys 66: 352-357, 2006.

7. Swain SM, Sorace RA, Bagley CS, Danforth DN Jr, Bader J, Wesley MN, Steinberg SM and Lippman ME: Neoadjuvant chemotherapy in the combined modality approach of locally advanced nonmetastatic breast cancer. Cancer Res 47: 3889-3894, 1987.

8. Mauri D, Pavlidis N and Ioannidis JP: Neoadjuvant versus adjuvant systemic treatment in breast cancer: A meta-analysis. J Natl Cancer Inst 97: 188-194, 2005.

9. Fisher B, Brown A, Mamounas E, Wieand S, Robidoux A, Margolese RG, Cruz AB Jr, Fisher ER, Wickerham DL, Wolmark N, et al: Effect of preoperative chemotherapy on local-regional disease in women with operable breast cancer: Findings from National Surgical Adjuvant Breast and Bowel Project B-18. J Clin Oncol 15: 2483-2493, 1997.

10. Julian TB, Patel N, Dusi D, Olson P, Nathan G, Jasnosz K, Isaacs $\mathrm{G}$ and Wolmark N: Sentinel lymph node biopsy after neoadjuvant chemotherapy for breast cancer. Am J Surg 182: 407-410, 2001.

11. van der Hage JA, van de Velde CJ, Julien JP, Tubiana-Hulin M, Vandervelden C and Duchateau L: Preoperative chemotherapy in primary operable breast cancer: Results from the European Organization for Research and Treatment of Cancer trial 10902. J Clin Oncol 19: 4224-4237, 2001.

12. Mieog JS, van der Hage JA and van de Velde CJ: Neoadjuvant chemotherapy for operable breast cancer. Br J Surg 94: 1189-1200, 2007.

13. Bloom HJ and Richardson WW: Histological grading and prognosis in breast cancer; a study of 1409 cases of which 359 have been followed for 15 years. Br J Cancer 11: 359-377, 1957.

14. Goldhirsch A, Winer EP, Coates AS, Gelber RD, Piccart-Gebhart M, Thürlimann B, Senn HJ, Albain KS, Andre F, Bergh J, et al; Panel members: Personalizing the treatment of women with early breast cancer: Highlights of the St Gallen International Expert Consensus on the Primary Therapy of Early Breast Cancer 2013. Ann Oncol 24: 2206-2223, 2013.

15. Abramoff MD, Magalhães PJ and Ram SJ: Image processing with ImageJ. Biophotonics Int 11: 36-42, 2004.

16. Ogston KN, Miller ID, Payne S, Hutcheon AW, Sarkar TK, Smith I, Schofield A and Heys SD: A new histological grading system to assess response of breast cancers to primary chemotherapy: Prognostic significance and survival. Breast 12: 320-327, 2003.

17. Rajan R, Poniecka A, Smith TL, Yang Y, Frye D, Pusztai L, Fiterman DJ, Gal-Gombos E, Whitman G, Rouzier R, et al: Change in tumor cellularity of breast carcinoma after neoadjuvant chemotherapy as a variable in the pathologic assessment of response. Cancer 100: 1365-1373, 2004. 